\title{
A New Active Contour Method based on Elastic Interaction
}

\author{
Yang Xiang $\dagger$, Albert C.S. Chung $\ddagger$ and Jian Ye $\nmid$ \\ $\dagger$ Department of Mathematics and $†$ Department of Computer Science, \\ The Hong Kong University of Science and Technology, Clear Water Bay, Hong Kong.
}

\begin{abstract}
Image segmentation is defined as partitioning an image into non-overlapping regions based on the intensity or texture. The active contour methods provide an effective way for segmentation, in which the boundary of an object (usually with large image gradient value) is detected by an evolving curve. But, these methods have limitations due to the fact that real images may have objects with complex geometric structures and shapes, and are often corrupted by noise. Developing more robust and accurate active contour methods has been an active research area since the idea of the methods was proposed. In this paper, we propose a new active contour method and apply the method to medical image segmentation. This new method uses a long-ranged interaction between image boundaries and the moving curves, which is inspired by the elastic interaction between line defects in solids (dislocations). The new method is more efficient and effective, especially in detecting thin, weak and blurred structures such as the images of blood vessels.
\end{abstract}

\section{Introduction}

Image segmentation is defined as partitioning an image into non-overlapping regions based on the intensity or texture. The active contour method for image segmentation was proposed in late 1980's [7]. In this method, a curve is evolved towards the object boundary under a force, until it stops at the boundary. In the classical active contour methods [7], the curve moves to minimize the energy

$$
\mathcal{E}(l)=\int_{l}\left(\frac{1}{2} \alpha\left|l^{\prime}(s)\right|+\frac{1}{2} \beta\left|l^{\prime \prime}(s)\right|-\lambda|\nabla I(l(s))|^{2}\right) d s
$$

where $l(s)$ represents a parameterized curve, $I(x, y)$ is the image gray-level function, and constants $\alpha, \beta, \lambda>0$. The first two terms in the energy functional smooth the curve. The third term attracts the curve to the object boundary, where the value of image gradient is large. The dynamics of the curve is given by the Euler-Lagrange equation

$$
l_{t}(s)=\alpha l^{\prime \prime}(s)-\beta l^{\prime \prime \prime \prime}(s)+\lambda \nabla|\nabla I(l(s))|^{2} .
$$

The image function $I(x, y)$ can be replaced by its smoothed version $G_{\sigma}(x, y) * I(x, y)$, where $G_{\sigma}(x, y)$ is a twodimensional Gaussian function with zero mean and standard deviation $\sigma$, and the operator $*$ is the convolution operator. Later, a constant force (balloon force) was added in the normal direction of the curve to accelerate the motion of the curve and increase the capture range [4]. In [2, 8], the level set framework [9] was used to handle the topological changes such as merging or splitting of the moving curve. The dynamic equation can be summarized as

$$
\phi_{t}=g(\nabla I)\left(\nabla \cdot\left(\frac{\nabla \phi}{|\nabla \phi|}\right)+\nu\right)|\nabla \phi|,
$$

where $\phi(x, y)$ is the level set function whose zero level set represents the curve. The terms before $|\nabla \phi|$ form the velocity of the curve in its normal direction. The first term in the bracket is the effect of the curvature of the curve, which smooths and shortens the curve. The second term in the bracket is a constant $\nu$, which corresponds to the balloon force mentioned above, making the curve expand or shrink depending on its sign. The function $g(\nabla I(x, y))$ is chosen such that it is very small at the boundary, where the value of image gradient is large, so that the velocity of the curve is small and the curve will stop there. One choice of $g(\nabla I(x, y))$ is $g(\nabla I(x, y))=1 /\left(1+\mid \nabla G_{\sigma}(x, y) *\right.$ $\left.\left.I(x, y)\right|^{p}\right), \quad p \geq 1$. There are some modifications to the level set formulation, stated in Eq. (3), rewriting the right-hand-side as the minimization of an energy $[3,14]$ which gives some additional terms to attract the curve to the boundary from its both sides. However, for these methods, without the balloon force, the capture range is short and the curve cannot reach the narrow concave parts of the boundary. This is because the effect of $\nabla I(x, y)$, defined by $\nabla|\nabla I(x, y)|^{2}$ in Eq.(2) or $g(\nabla I(x, y))$ in Eq.(3), is localized near the boundary. While with the balloon force, there is a limitation that the balloon force cannot make some parts of curve shrink while other parts of the curve expand, so the 
initial curve must be placed entirely outside or inside the object to be detected.

To solve the problem of these methods, $\mathrm{Xu}$ and Prince [16] proposed the gradient vector flow (GVF) method. In this method, the attractive force near the object boundary $\nabla|\nabla I(l(s))|^{2}$ (see Eq.(2)) is extended to the whole computational region by diffusion. They obtained a force field $\mathbf{v}=(u, v)$ from an image by minimizing the energy

$$
\mathcal{E}=\iint \mu\left(u_{x}^{2}+u_{y}^{2}+v_{x}^{2}+v_{y}^{2}\right)+|\nabla f|^{2}|\mathbf{v}-\nabla f|^{2} d x d y
$$

where $f=|\nabla I(x, y)|^{2}, \nabla f=\nabla|\nabla I(x, y)|^{2}$ represents the attractive force to the boundary, and $\mu$ is the diffusion constant. In this equation, near the object boundary, $|\nabla f|$ is large, the second term dominates and the minimization gives $\mathbf{v}=\nabla f$; while away from the boundary, $|\nabla f|$ is small and thus the second term is small, the energy is dominated by the diffusion term, which means the force $\mathbf{v}$ is extended smoothly from its value near the object boundary. Therefore, the capture range is longer than that of the previous methods and there is no need to place the initial curve entirely inside or outside the object. However, the initial curve must be placed near the object boundary to be detected; otherwise it might be attracted to a boundary of a wrong object or a wrong part of the boundary. Besides, it is still not effective for detecting narrow concave boundaries, even with the modifications made afterwards [11] (see Fig.5(a)).

In this paper, we propose a new active contour method, which is inspired by the elastic interaction between line defects (dislocations) in solids [5, 15]. There is a longrange attractive interaction between two dislocation lines with different directions. The interaction is proportional to $1 / r$ for two long straight parallel dislocations, where $r$ is the distance between them. Using a similar idea for twodimensional image segmentation, we shall define a longrange attractive force generated by the object boundary and acting on the moving curve, and use the level set framework to handle the topological changes that might occur during the evolution of the curve. In Sec.2, we will present the formulation and numerical implementation for this new method. In Sec.3, we will show by examples that the new method is more efficient and effective, especially in detecting thin, weak and blurred structures such as the images of blood vessels.

\section{Methodology}

\subsection{Attractive force based on elastic interaction}

We use the level set framework [9] to represent and evolve the curve. Let $\phi(x, y)$ be the level set function whose zero level set represents the moving curve. The evolution equation of the level set function is

$$
\phi_{t}=v|\nabla \phi|
$$

where $v(x, y)$ is the normal velocity of the curve extended to the whole space.

We now define the velocity field using three-dimensional formulations. Considering a parameterized curve $\gamma(s)$ in the three-dimensional space, we define an energy associated with $\gamma(s)$

$$
\mathcal{E}(\gamma)=\min \int \frac{1}{2}\left(w_{1}^{2}+w_{2}^{2}+w_{3}^{2}\right) d x d y d z
$$

subject to the constraint

$$
\nabla \times \mathbf{w}=\delta_{2}(\gamma) \mathbf{t}
$$

where $\mathbf{w}=\left(w_{1}, w_{2}, w_{3}\right)$ is a function defined in the threedimensional space, $\mathbf{t}$ is the line direction of $\gamma(s), \delta_{2}(\gamma)$ is the Dirac delta-function of $\gamma(s)$ which is zero anywhere except on $\gamma(s)$. In Eq.(7), the operator $\nabla$ is a threedimensional operator. This convention will be used in and only in this paragraph; in other paragraphs, it is still a two-dimensional operator. After taking variation, we have Eq.(7) and

$$
\nabla \cdot \mathbf{w}=0
$$

This is a simplified version of the elasticity system associated with dislocations in solids [5]. This system is also similar to those for vortices in superconductivity [13] and fluid dynamics [1]. This system for $\mathbf{w}$ can be solved analytically $[1,5]$, which is

$$
\mathbf{w}(x, y, z)=-\frac{1}{4 \pi} \int_{\gamma} \frac{\mathbf{r} \times d \mathbf{l}}{r^{3}},
$$

where $\mathbf{r}=(x-x(s), y-y(s), z-z(s))$ is the vector between the point $(x, y, z)$ and a point $(x(s), y(s), z(s))$ on $\gamma(s)$, and $r=\sqrt{(x-x(s))^{2}+(y-y(s))^{2}+(z-z(s))^{2}}$ is the distance between them. The solution is singular on $\gamma(s)$ and as a result, the energy $\mathcal{E}(\gamma)$ is also singular. The singularities can be smeared out if we use a smeared deltafunction in Eq.(7). Similar to the properties of dislocations [5], if $\gamma(s)$ is a single loop or line, the energy is proportional to its length; if $\gamma(s)$ consists of multiple loops or lines, besides the energy proportional to the length, there is another contribution to energy due to the interaction of different loops or lines. The interaction energy of two loops depends on their line directions, their relative position and their length. If we allow $\gamma(s)$ to move to minimize $\mathcal{E}(\gamma)$, the Euler-Lagrange equation is

$$
\gamma_{t}=\mathbf{w} \times \mathbf{t}
$$


Under this velocity field, the energy is reduced by shrinking of $\gamma(s)$, attraction and annihilation of segments of $\gamma(s)$ with opposite directions, and repulsion of segments with same direction.

Now we consider our segmentation problem. We put the image in the $z=0$ plane and use the above formulation to compute the force acting on the moving curve. If $I(x, y)$ is the image gray-level function, the direction of the object boundary is defined as

$$
\begin{aligned}
\mathbf{t} & =\frac{1}{\left|\nabla\left(G_{\sigma} * I\right)\right|}\left(\frac{\partial\left(G_{\sigma} * I\right)}{\partial x}, \frac{\partial\left(G_{\sigma} * I\right)}{\partial y}, 0\right) \times(0,0,1), \\
& =\frac{1}{\left|\nabla\left(G_{\sigma} * I\right)\right|}\left(\frac{\partial\left(G_{\sigma} * I\right)}{\partial y},-\frac{\partial\left(G_{\sigma} * I\right)}{\partial x}, 0\right) .
\end{aligned}
$$

Under this definition, if the image of an object has a stronger intensity than the background, the direction of the object boundary is counterclockwise; and is clockwise vice versa. The direction of the moving curve, i.e., the zero level contour of the level set function $\phi(x, y)$, is defined similarly, with $G_{\sigma} * I$ replaced by $\phi$ in the above equation. The level set function is chosen such that the moving curve has an opposite direction with the object boundary. As a result, the moving curve is attracted to the object boundary under the force generated by the object boundary. The delta-function in Eq.(7) can be approximated by $\left|\nabla\left(G_{\sigma} * I\right)\right| \delta(z)$, where $\delta(z)$ is the one-dimensional Dirac delta-function of $z$. As such, for Eq.(7)

$$
\delta_{2}(\gamma) \mathbf{t}=\left(\frac{\partial\left(G_{\sigma} * I\right)}{\partial y},-\frac{\partial\left(G_{\sigma} * I\right)}{\partial x}, 0\right) \delta(z) .
$$

In summary, w can be obtained by solving Eqs. (7), (8) and (12). The solution is given by Eq.(9), where $\gamma$ is the object boundary. Using Eq.(9), it can be verified that $w_{1}(x, y, 0)=$ $w_{2}(x, y, 0)=0$. As such, by Eq.(10), the velocity (force) generated by the object boundary and acting on the moving curve is

$$
v=w_{3}(x, y, 0) \nabla \phi /|\nabla \phi| .
$$

We can add a small curvature term to smooth the moving curve, then the evolution equation can be written as

$$
\phi_{t}=\left(\mu \nabla \cdot\left(\frac{\nabla \phi}{|\nabla \phi|}\right)+w_{3}(x, y, 0)\right)|\nabla \phi|,
$$

where $\mu$ is a small constant.

It is not easy to compute $w_{3}$ from Eq.(9). We use the Fast Fourier Transform (FFT) to solve for $w_{3}$ from Eqs.(7), (8) and (12). Assume that the image size is $[-\pi, \pi]^{2}$, and is placed in the $z=0$ plane of a three-dimensional computation cell $[-\pi, \pi]^{3}$ with periodic boundary conditions in $x, y$ and $z$ directions. The solution of the Eqs.(7), (8) and (12) is

$$
=\sum_{m, n} \frac{\sqrt{m^{2}+n^{2}}}{2} \cdot \frac{e^{\pi \sqrt{m^{2}+n^{2}}}+e^{-\pi \sqrt{m^{2}+n^{2}}}}{e^{\pi \sqrt{m^{2}+n^{2}}}-e^{-\pi \sqrt{m^{2}+n^{2}}}} \cdot d_{m n} e^{i m x} e^{i n y},
$$

where $m^{2}+n^{2} \neq 0$, and $\left\{d_{m n}\right\}$ are the two-dimensional Fourier coefficients of the function $G_{\sigma} * I(x, y)$, i.e.,

$$
G_{\sigma} * I(x, y)=\sum_{m, n} d_{m n} e^{i m x} e^{i n y} .
$$

We can see that even though we use three-dimensional formulations, the effect in $z$ direction can be computed analytically, and $w_{3}$ can be obtained by FFT only in $x y$ space. We only need to perform an FFT using Eq.(16) and an inverse FFT using Eq.(15). Therefore it is still a two-dimensional problem and is very efficient in terms of computation. If the image size is not $(2 \pi)^{2}$, there will be a constant coefficient in Eq.(15), which does not change the dynamics of the moving curve. In all cases, a normalized velocity field,

$$
w_{3}(x, y, 0) /\left|w_{3}(x, y, 0)\right|,
$$

can be used to accelerate the evolution.

Regarding the numerical implementation for the evolution equation of the level set function $\phi$ (Eq.(14)), we use central difference for the curvature term, Godunov's scheme [10] combined with third order WENO derivative [6] for the term $w_{3}|\nabla \phi|$, and the forward Euler method in time. To reduce the numerical errors, a standard technique called reinitialization is used to maintain an accurate distance function near the zero level set of the level set function during its evolution [12].

\subsection{Noise removal and initialization}

In the evolution equation (Eq.(14)), there are two ways to remove noise and smooth the moving curve. The first way is to control the standard deviation $\sigma$ of the Gaussian function in Eqs. (16) and (15) when computing $w_{3}$. The second way is to control the coefficient $\mu$ of the curvature term. Large values of $\sigma$ and $\mu$ can remove noise effectively, but they can also over-smooth the object boundary. Small values of $\sigma$ and $\mu$ can keep sharp features of the objects, but is not very effective for noise removal. We combine them to remove the noise and keep the sharp features. This can be done efficiently by the following way. First note that the velocity field $w_{3}$ has different signs inside and outside an object. Without noise, the zero level contour of $w_{3}$ actually gives the boundary of the object. However, with noise present, which is the case for real images, large amount of noise is also kept. The zero level contours of $w_{3}$ computed from a large value of $\sigma$ and a small value of $\sigma$ can be combined (by taking either max or min of $w_{3}$ 's, depending on the sign of the object boundary) to get an approximation of the object with only very small amount of noise. The small amount of noise can be further removed by the evolution of the curve with only the curvature term in Eq.(14). Then the obtained curve is used as the initial curve, and is evolved using Eq.(14) with small values of $\sigma$ and $\mu$ to recover the object boundary accurately. 


\subsection{Outline of the algorithm}

For an image without noise, the contour is simply the zero level contour of the normalized velocity field, $w_{3} /\left|w_{3}\right|$. No evolution is needed.

For an image with noise, there are two major steps:

1. Noise removal and initialization

(1) Compute the normalized velocity field $f_{1}=w_{3} /\left|w_{3}\right|$ using a small $\sigma$;

(2) Compute the normalized velocity field $f_{2}=w_{3} /\left|w_{3}\right|$ using a big $\sigma$;

(3) Take $f=\max \left\{f_{1}, f_{2}\right\}$ (or min depending on the sign of the object boundary); and

(4) Evolve $f$ under the curvature flow for a few steps and assign the result function to be the initial value of $\phi$.

2. Evolution of $\phi$ using Eq.(14) with the velocity field $f_{1}$. Re-initialization is performed when necessary.

\section{Experimental Results}

We have applied the proposed method on the synthetic and real images and also performed comparison between our method and the gradient vector flow (GVF) method $[16,11]$ (The results using these GVF methods are similar in the comparison. The results shown are the results obtained using the modified GVF method with balloon force [11]). All methods were programmed in Fortran and run on a $2.8 \mathrm{GHz}$ PC with $256 \mathrm{MB}$ RAM.

\subsection{Synthetic Images}
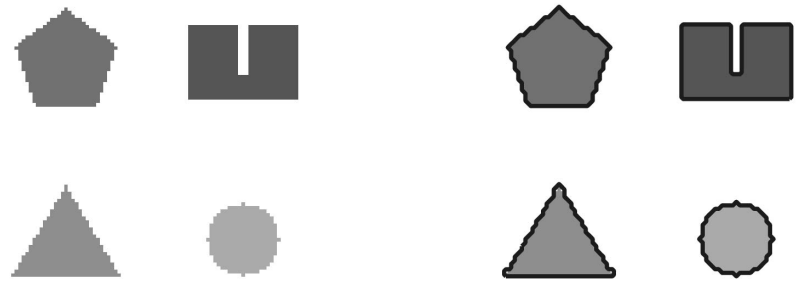

(a)

(b)

Figure 1. Multiple objects without noise. (a) Input image. (b) Contours found by our method.

All results in this section were obtained using a numerical mesh with the size of $128 \times 128$ pixels. The length was equal to 2 units. As such, the pixel width is $d x=d y=$ 2/128. Fig. 1 shows the results on an image with multiple objects, which have different intensity and topology, but without noise. The intensity values of the four objects were
$3 / 9,4 / 9,5 / 9$ and $6 / 9$, respectively. We set $\sigma=2.4 d x$. Fig.1(b) shows the zero level contours of the normalized velocity field, which was calculated by FFT using Eq.(15). It shows that our method is very efficient and no evolution is needed.

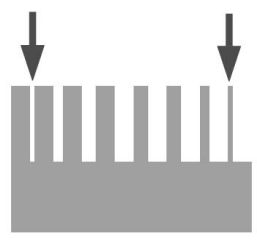

(a)

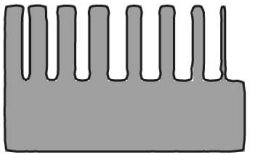

(b)
Figure 2. Thin concave and convex structures. (a) Input image. (b) Contour found by our method.

Fig.2 shows the experimental result on an image of an object which has very thin convex feature on its right and concave feature on its left, at which these features are pointed by the arrows, see Fig.2(a). Both features are only one pixel width. The zero level contour of the normalized velocity field $w_{3}$ using $\sigma=2.4 d x$ gave a good result, but on the tip of the one-pixel convex, the contour width was a little bit bigger than one pixel. To obtain a more accurate result, we used $\sigma=d x$ to calculate $w_{3}$. However, this caused numerical oscillation of the velocity field and generated some spurious contours away from the object. This numerical noise can be removed using the same way as for removing the image noise. As such, the zero level contour of the maximum of the two normalized velocity fields $w_{3}$ ( $\sigma=d x, 2.4 d x$ ) found the object accurately, as shown in Fig.2(b). Still, no evolution is needed.

These two examples show that our method can find the object accurately and efficiently (without evolution) if the image has no noise.

Using the image shown in Fig.1(a), Fig.3 shows the results of our method applied on the image corrupted by zero mean Gaussian noise with $\sigma=0.1$. First of all, we used $\sigma_{1}=2.4 d x$ and $\sigma_{2}=10 d x$ to compute the velocity fields $w_{3}$, respectively. The two velocity fields, after normalization, are denoted by $f_{1}$ and $f_{2}$, respectively. The zero level contours of $f_{1}$ are shown in Fig.3(b). The contours found the sharp features of the objects and a large amount of noise. The zero level contours of $f_{2}$ are shown in Fig.3(c). The contours only kept a small amount of noise but were oversmoothed. After taking the maximum of $f_{1}$ and $f_{2}$, better contours were obtained, as shown in Fig.3(d). After a few steps of smoothing using the mean curvature flow, we got more smooth initial contours without noise, as shown 


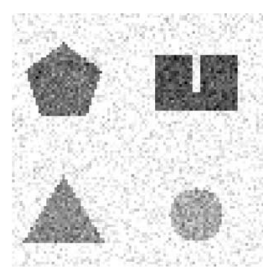

(a)

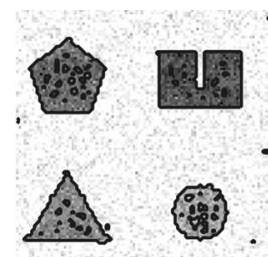

(d)

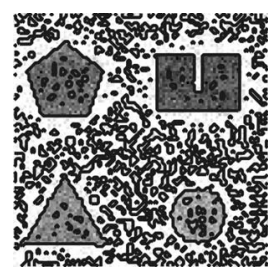

(b)

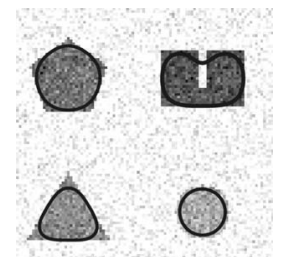

(e)

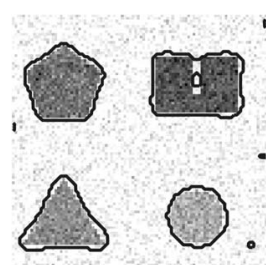

(c)

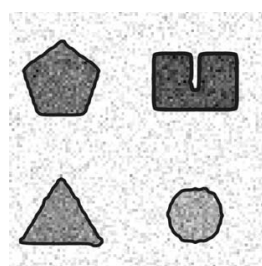

(f)
Figure 3. Multiple objects with Gaussian noise. (a) Input image. (b) Contours of $f_{1}=0$. (c) Contours of $f_{2}=0$. (d) Contours of $\max \left\{f_{1}, f_{2}\right\}=0$. (e) Contours after smoothing. (f) Final result after evolution.

in Fig.3(e). These initial contours were then evolved using Eq.(14) under the velocity field $f_{1}$ with $\mu=0.015$ to recover the sharp features of the objects, as shown in Fig.3(f).

This example shows that our method is also efficient and effective for an image with noise, in which an initial contour close to the object can be first calculated efficiently, then the detailed features are recovered quickly by further evolution of the contour.

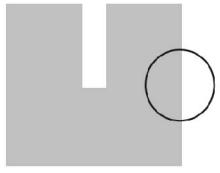

(a)

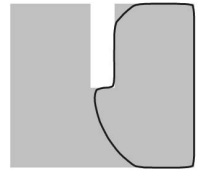

(b)

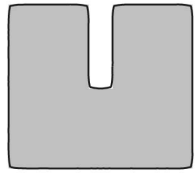

(c)
Figure 4. Contour found from an initial contour not close to the object using Eq.(14). (a) Initial contour and object. (b) Intermediate result. (c) Final result.

In the next example, we show the ability of our method to recover the detailed features of the objects by evolving an initial contour that is not close to the object boundary. Note that it is only to mimic the second stage of our method for images with noise. If an image itself has no noise, objects in the image can be found efficiently by calculating the zero level contour of the normalized velocity field. The result is shown in Fig.4. We started from a small circle intersecting with the right hand side of a $U$ shape object, as shown in Fig.4(a), and evolved it using Eq.(14) with $\sigma=2.4 d x$ and $\mu=0.01$. The contour outside the object was attracted to the object boundary. The contour inside the object was pushed far away to touch the another side of the object boundary. An intermediate result after several steps is shown in Fig.4(b). Finally, the contour converged to the object boundary, as shown in Fig.4(c).

Fig.5(a) shows the result obtained using the GVF method. The GVF method was applied on the image shown in Fig.2(a). The initial contour started from a circle enclosing the object. It is observed that the final contour found by the GVF method cannot correctly detect the thin convex and concave features. It is because the GVF method must smooth the gradient values on the boundary in order to get an extended force field. We have also applied the GVF method on the same U shape object with the same initial contour as shown in Fig.4(a). The contour shrank and finally disappeared. It was because both sides of the contour were attracted to the right hand side of the object boundary. This can be seen from the force field near the object boundary and the initial contour plotted in Fig.5(b). It illustrates that the GVF method is dependent on the contour initialization. In contrast, the evolution stage of our method has less sensitivity to the initialization.

From these examples, it can be seen that our method is more efficient and effective than the GVF method. The computation times for our method (Fig.2b) and the GVF method (Fig.5a) were 0.49 and 141.96 seconds respectively.

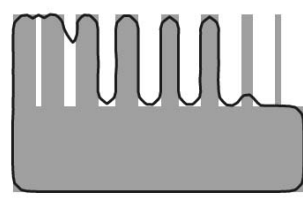

(a)

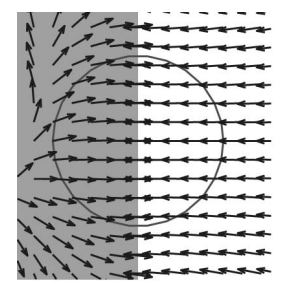

(b)
Figure 5. GVF method for the images in Figs. 2 and 4. (a) GVF method for the thin concave and convex structures as shown in Fig.2(a): final result. (b) GVF method for the U shape object in Fig.4(a): force field near the object boundary and the initial contour.

\subsection{Real Image}

In Fig.6, we applied our method on a real image of blood vessels. The image was acquired and obtained from the 


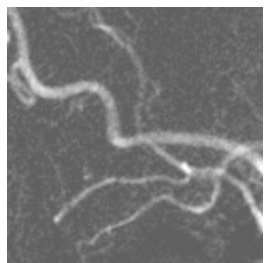

(a)

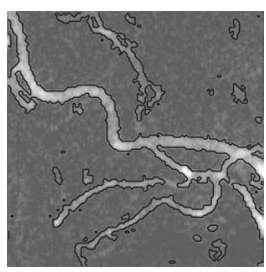

(d)

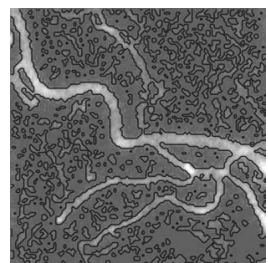

(b)

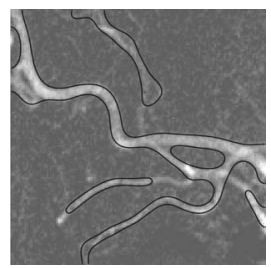

(e)

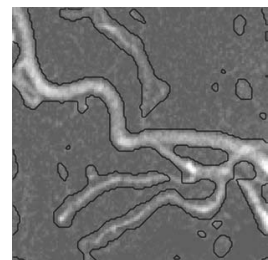

(c)

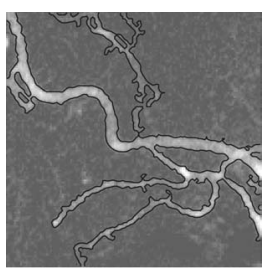

(f)
Figure 6. Blood vessels. (a) Input image. (b) Contours of $f_{1}=0$. (c) Contours of $f_{2}=0$. (d) Contours of $\min \left\{f_{1}, f_{2}\right\}=0$. (e) Contours after smoothing. (f) Final result.

Prince of Wales Hospital, Hong Kong. The image size was $128 \times 128$ pixels. This image is more noisy and has more complex structures. First of all, we used $\sigma_{1}=2.4 d x$ and $\sigma_{2}=10 d x$ to compute the velocity fields $w_{3}$, respectively. The two velocity fields, after normalization, are denoted by $f_{1}$ and $f_{2}$, respectively. The zero level contours of $f_{1}$ are shown in Fig.6(b). The contours found sharp features of the blood vessels, as well as a large amount of noise. The zero level contours of $f_{2}$ are shown in Fig.6(c). The contours kept only a small amount of noise but were over-smoothed. After taking the minimum of $f_{1}$ and $f_{2}$, better contours were obtained, as shown in Fig.6(d). After a few steps of smoothing using the mean curvature flow, we got more smooth initial contours as shown in Fig.6(e). These initial contours were then evolved under the velocity field $f_{1}$. After evolution, some small holes inside the contours were lost. We took the minimum of the final state of the level set function $\phi$ and $f_{1}$. Then those small holes were recovered (for example, see two small holes near the left edge of the image, as shown in Fig.6(f)). The final result is shown in Fig.6(f). It is noted that some parts of the contours are nearly disconnected because the intensity contrast is very low.

\section{Summary and Discussions}

We proposed a new active contour method and have applied the method to medical image segmentation. The new method is based on a long-range elastic interaction between the object boundary and the moving contour. The interaction force can be calculated efficiently by the Fast Fourier
Transform (FFT). For an image without noise, objects can be found by the zero level of this force without evolution in time. For an image with noise, an initial contour very closed to the object boundaries is first computed efficiently, and then detail features of objects are recovered after a shorttime evolution. Examples show that this new method is more efficient and effective, especially in detecting thin, weak and blurred structures such as the images of blood vessels.

\section{References}

[1] G. Batchelor. Introduction to Fluid Mechanics. Cambridge Univ. Press, Cambridge, 1967.

[2] V. Caselles, F. Catte, T. Coll, and F. Dibos. A geometric model for active contours in image processing. Numer. Math., (66):1-31, 1993.

[3] V. Caselles, R. Kimmel, and G. Sapiro. Geodesic active contours. Int. J. Comput. Vision, (22):61-79, 1997.

[4] L. Cohen. On active contour models and balloons. Computer Vision, Graphics, and Image Processing: Image Understanding, (53):211-218, 1991.

[5] J. Hirth and J. Lothe. Theory of Dislocations, 2nd edition. John Wiley, New York, 1982.

[6] G. Jiang and D. Peng. Weighted eno schemes for hamiltonjacobi equations. J. Sci. Comput., (21):2126-2143, 2000.

[7] M. Kass, A. Witkin, and D. Terzopoulos. Snakes: Active contour models. Int. J. Comput. Vision, (1):321-331, 1988.

[8] R. Malladi, J. Sethian, and B. Vemuri. Shape modeling with front propagation: A level set approach. IEEE Trans. Pattern Anal. Machine Intell., (17):158-175, 1995.

[9] S. Osher and J. Sethian. Fronts propagating with curvaturedependent speed: algorithms based on hamilton-jacobi formulations. J. Comput. Phys., (79):12-49, 1988.

[10] S. Osher and C. Shu. High-order essentially nonoscillatory schemes for hamilton-jacobi equations. SIAM J. Numer. Anal., (28):907-922, 1991.

[11] N. Paragios, O. Mellina-Gottardo, and V. Ramesh. Gradient vector flow fast geometric active contours. IEEE Trans. Pattern Anal. Machine Intell., (26):402-407, 2004.

[12] D. Peng, B. Merriman, S. Osher, H. Zhao, and M. Kang. A pde-based fast local level set method. J. Comput. Phys., (155):410-438, 1999.

[13] L. Pismen. Vortices in nonlinear fields. Oxford Univ. Press, New York, 1999.

[14] K. Siddiqi, Y. Lauziere, A. Tannenbaum, and S. Zucker. Area and length minimizing flows for shape segmentation. IEEE Trans. Image Processing, (7):433-443, 1998.

[15] Y. Xiang, L. Cheng, D. Srolovitz, and W. E. A level set method for dislocation dynamics. Acta Mater., (51):54995518, 2003.

[16] C. Xu and J. Prince. Snakes, shapes, and gradient vector flow. IEEE Trans. Image Processing, (7):359-369, 1998. 